Supporting Information for

\title{
Transient Diffusive Interactions with a Protein Crowder Affect Aggregation Processes of Superoxide Dismutase $1 \beta$-Barrel
}

Naoto Iwakawa ${ }^{\dagger}$, Daichi Morimoto ${ }^{\dagger}$,Erik Walinda ${ }^{\ddagger}$, Sarah Leeb $^{\S}$, Masahiro Shirakawa ${ }^{\dagger}$, Jens Danielsson ${ }^{\S}$, and Kenji Sugase ${ }^{*} \dagger$

'Department of Molecular Engineering, Graduate School of Engineering, Kyoto University, Kyoto-Daigaku Katsura, Nishikyo-ku, Kyoto 615-8510, Japan

*Department of Molecular and Cellular Physiology, Graduate School of Medicine, Kyoto University, Yoshida Konoe-cho, Sakyo-ku, Kyoto 606-8501, Japan

${ }^{\S}$ Department of Biochemistry and Biophysics, Arrhenius Laboratories of Natural Sciences, Stockholm University, 10691 Stockholm, Sweden

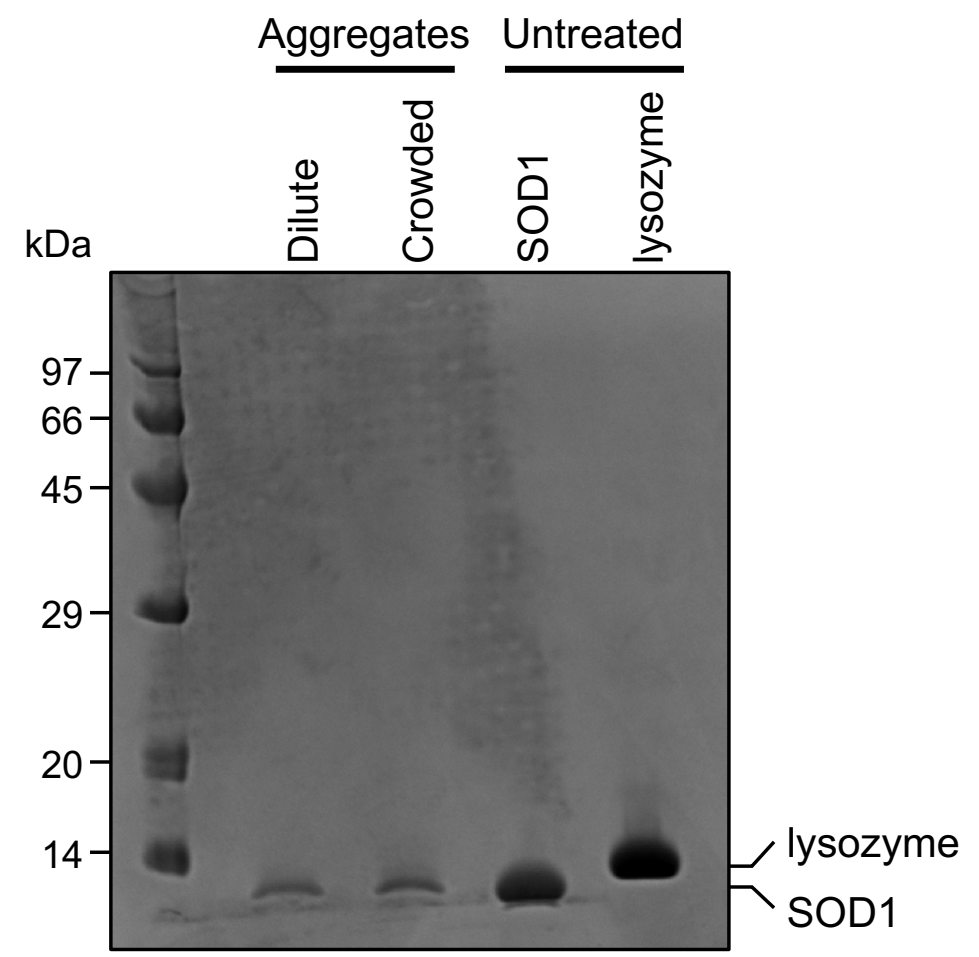

Figure S1. Components of aggregates generated in the dilute and crowded conditions.

SDS-PAGE analysis of aggregates (fibrils) generated in the dilute and crowded conditions. Untreated SOD1 (11 kDa) and lysozyme $(14 \mathrm{kDa})$ were run in the right lanes. 

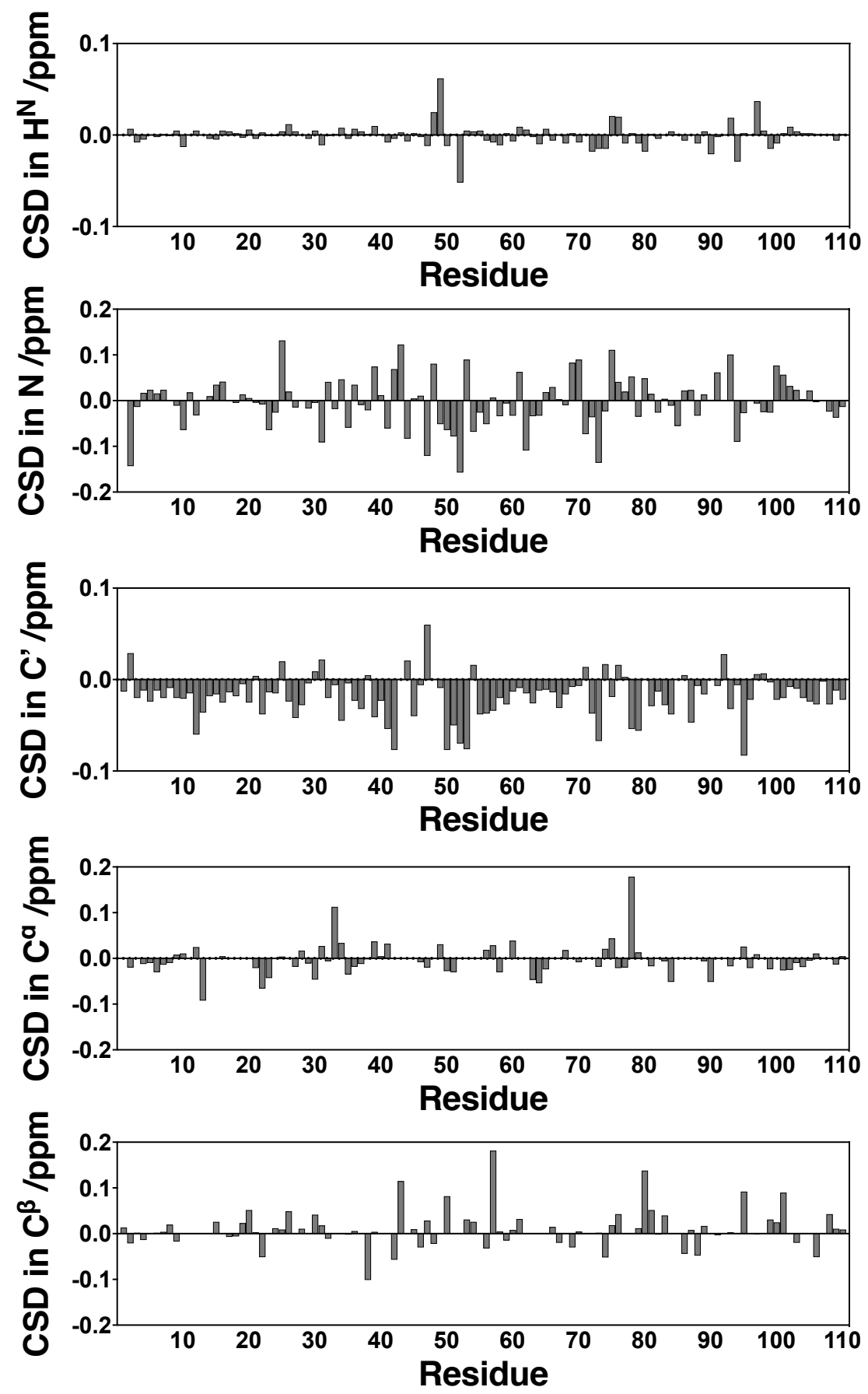

Figure S2. Chemical shift changes of native SOD1 caused by the addition of lysozyme.

Shown are chemical shift differences $(\mathrm{CSD})$ in $\mathrm{H}^{\mathrm{N}}, \mathrm{N}, \mathrm{C}^{\prime}, \mathrm{C}^{\alpha}$, and $\mathrm{C}^{\beta}$ atoms (from top to bottom) due to addition of the protein crowder lysozyme. 

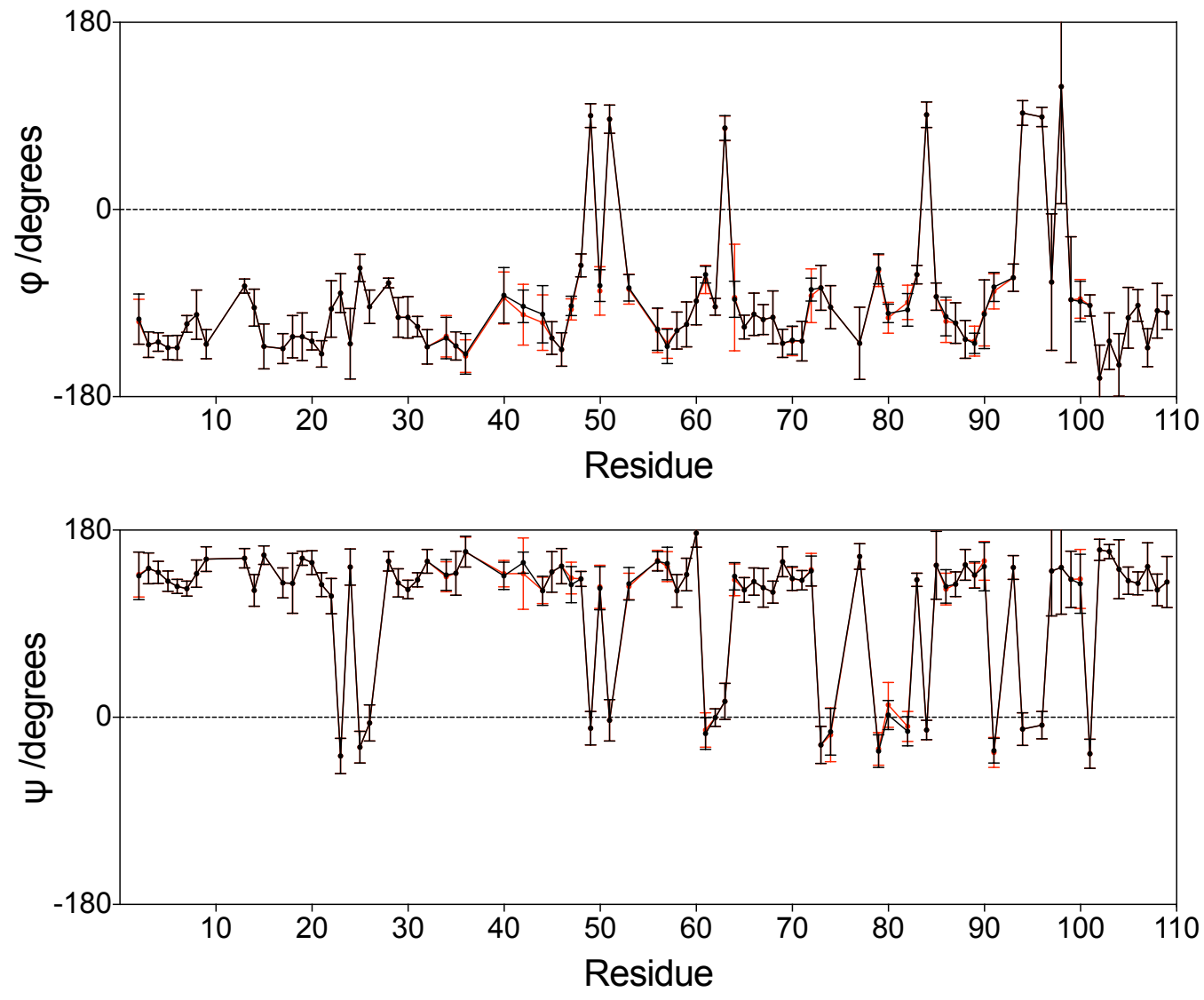

Figure S3. Effects of lysozyme on the dihedral angles of SOD1.

Shown are dihedral angles $\varphi$ (upper) and $\psi$ (lower) in the dilute (black) and crowded (red) environments as predicted from the experimentally measured chemical shifts using TALOS $+^{1}$. Error bars represent the estimated standard deviations of the prediction errors in $\varphi$ and $\psi$. 


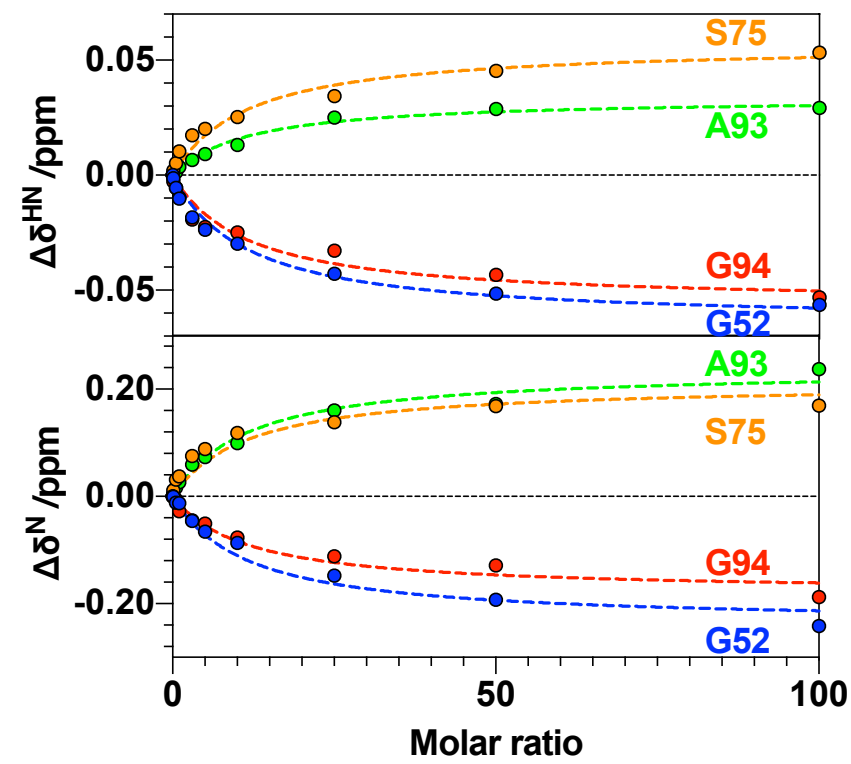

Figure S4. Binding of lysozyme to native SOD1 as determined by NMR titration experiments.

Amide proton $\left(\mathrm{H}^{\mathrm{N}}\right.$, upper) and amide nitrogen $\left({ }^{15} \mathrm{~N}\right.$, lower) chemical shift changes of representative residues are plotted (filled circles) against the molar ratio of lysozyme to SOD1 with fitting curves (dashed lines) to equation 2 (see Methods). Gly52, blue; Ser75, orange; Ala93, green; Gly94, red. Initial concentrations (i.e., of the stock solutions before titration) of the analyte (SOD1) and titrant (lysozyme) were $0.2 \mathrm{mM}$ and $9.78 \mathrm{mM}$, respectively.
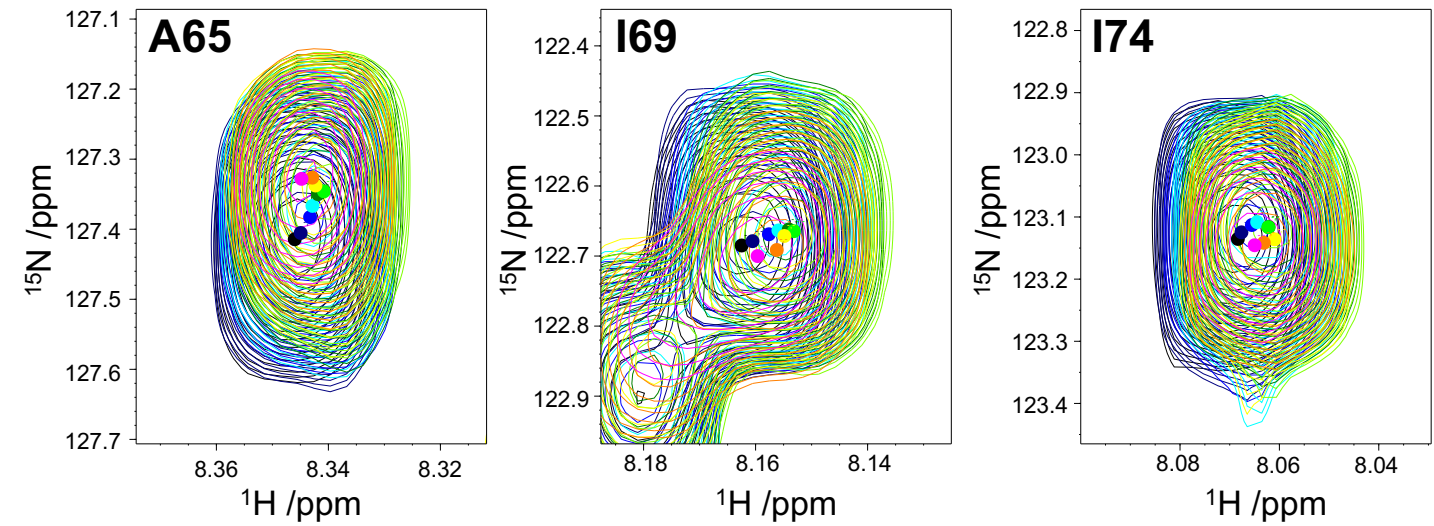

Figure S5. Observation of unusual chemical shift change pattern for the interaction of denatured SOD1 with lysozyme.

Shown are enlarged views of ${ }^{1} \mathrm{H}^{-15} \mathrm{~N}$ HSQC spectra of (unfolded) SOD1 ${ }^{\Delta \mathrm{VV} \Delta \mathrm{VIV} 29 \mathrm{AI} 35 \mathrm{~A}}$ highlighting Ala65 (left), Ile69 (middle), and Ile74 (right) in the absence (black) and presence of 0.1 (navy), 0.5 (blue), 1 (cyan), 3 (green), 5 (light green), 10 (yellow), 25 (orange), and 50 (magenta) equivalents of lysozyme. The position of the center of each cross-peak in the superimposed spectra is highlighted by the colored filled circles. 

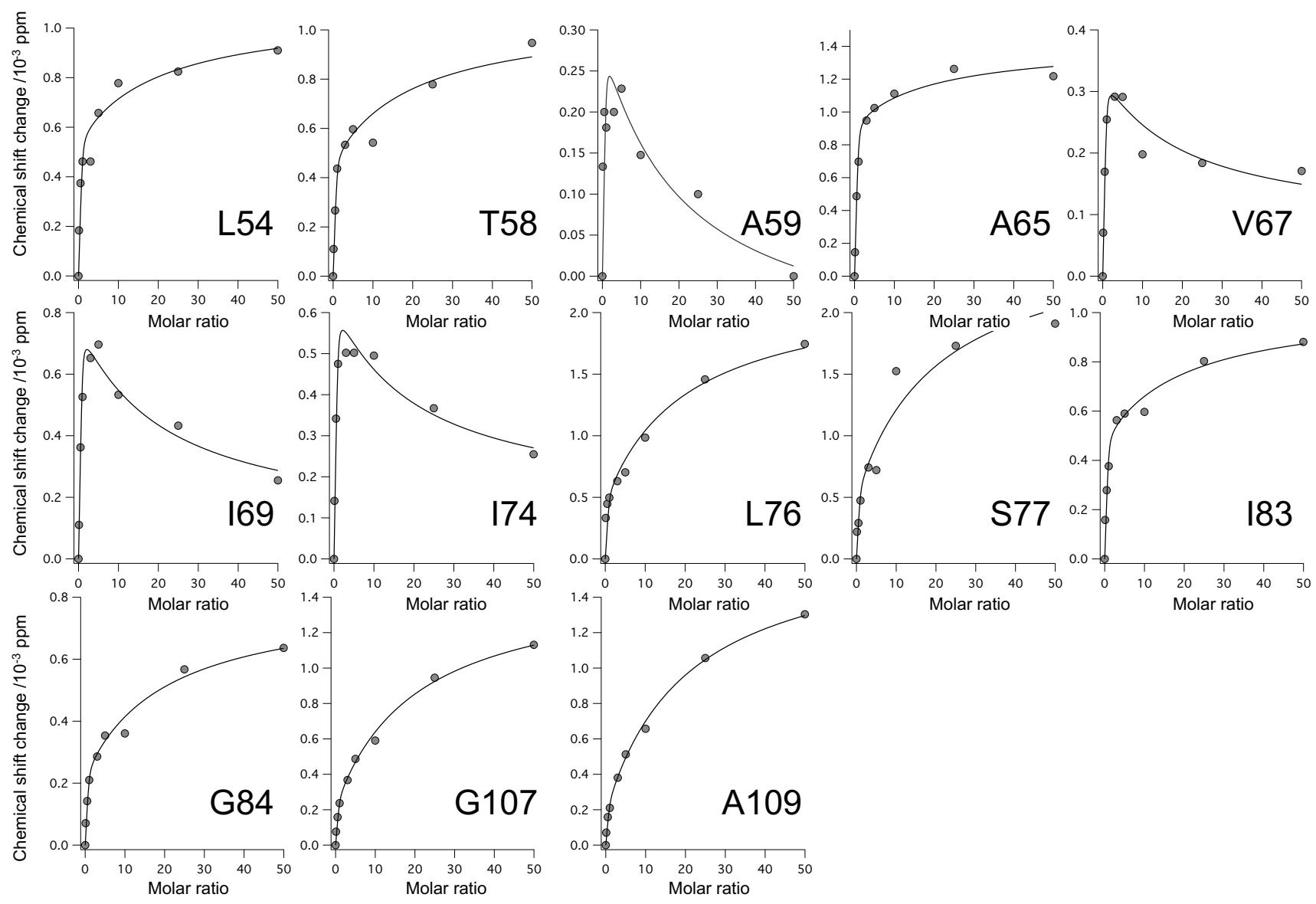

Figure S6. Chemical shift changes of denatured SOD1 upon binding to lysozyme.

Average chemical shift changes are plotted (filled circles) with fitting curves to a two-site binding model. Residue number and amino acid type (one-letter code) are displayed in each graph. Val67 is omitted from the global analysis (to determine dissociation constants) due to the poor agreement between individual and global fits. 


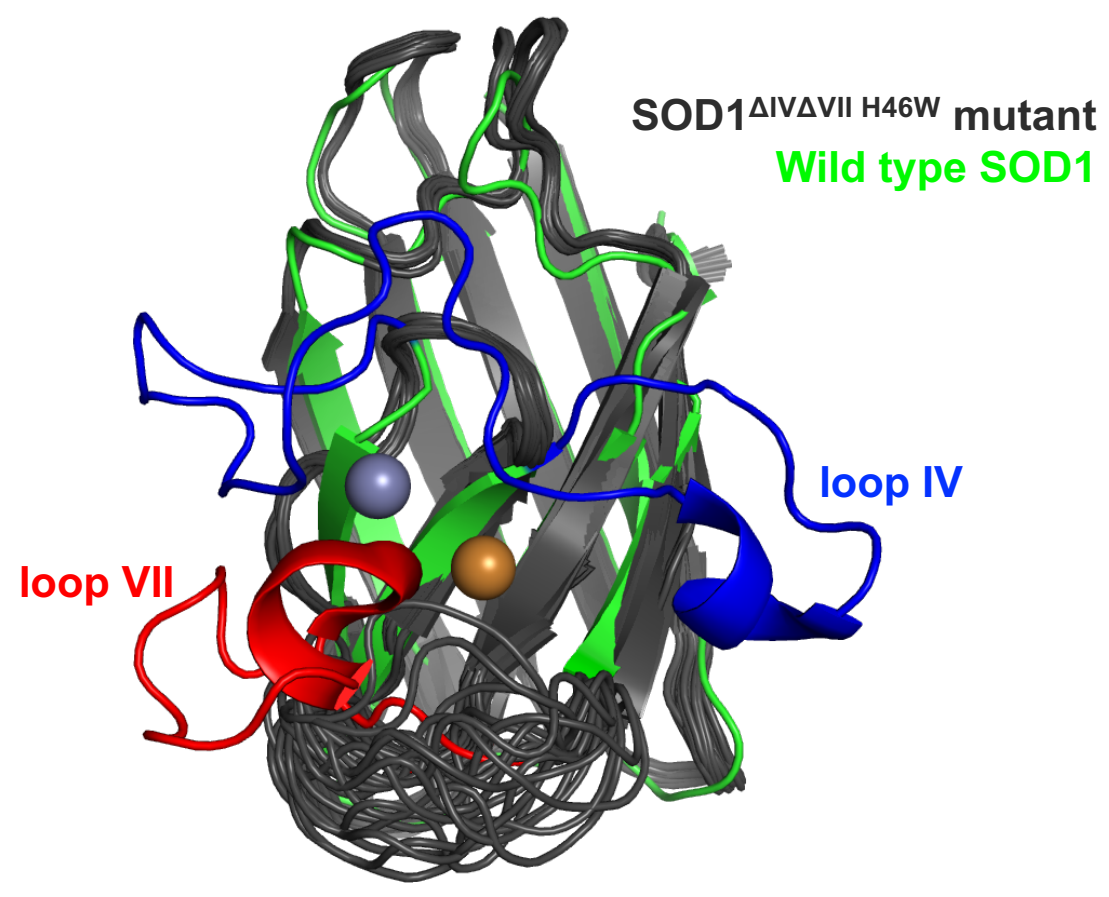

Figure S7. Comparison of the determined SOD1 $\beta$-barrel solution structure with the crystal structure of wild-type SOD1.

The crystal structure of wild-type SOD1 (PDB ID: 1HL5, green) is shown as an overlay with the solution structure (ensemble of 20 minimum energy structures) of the SOD1 $\beta$-barrel mutant (SOD1 ${ }^{\Delta \mathrm{IV} \Delta \mathrm{VII} H 46 \mathrm{~W}}$ ) that we determined in the absence of lysozyme (black). Two loops connecting strands $\beta 4$ and $\beta 5$ (loop IV), $\beta 7$ and $\beta 8$ (loop VII), which are truncated in the SOD1 $\beta$-barrel mutant, are shown in blue and red, respectively, and zinc and copper ions are shown as gray and brown spheres, respectively. Except for the regions adjacent to the truncated loops, no significant structural differences were observed. 

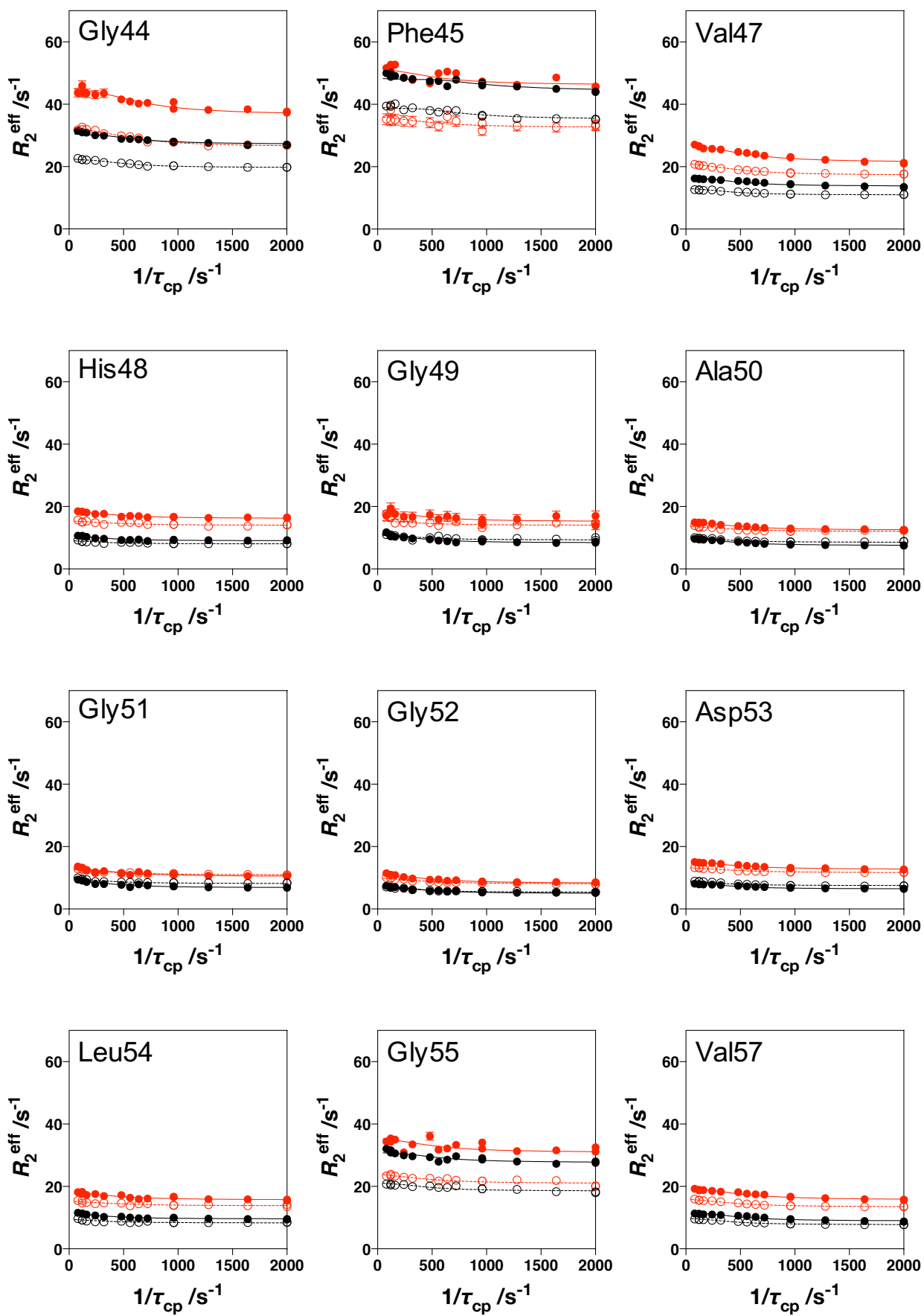

Figure S8. $R_{2}$ relaxation dispersion profiles of residues in global analysis of the $\mathbf{N}-\mathbf{N}^{*}$ transition.

Data corresponding to the dilute and crowded environments are colored in black and red, respectively. Solid and dashed lines represent best-fit curves of the data acquired at $18.8 \mathrm{~T}$ (filled circles) and $14.1 \mathrm{~T}$ (open circles), respectively. The residue number and amino acid type (three-letter code) are displayed in the upper left of each graph. 


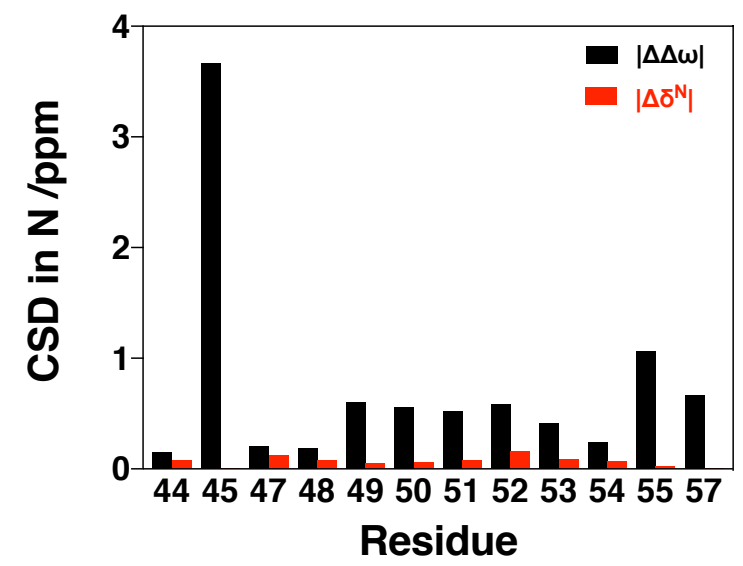

Figure S9. ${ }^{15} \mathrm{~N}$ chemical shift changes of the native and excited states in the presence of lysozyme.

Shown are changes in $\Delta \omega(|\Delta \Delta \omega|)$ (black) and in the chemical shift of the native state $\left(\left|\Delta \delta^{\mathrm{N}}\right|\right)$ (red) as determined by $R_{2}$ relaxation dispersion experiments (Figure 5) and ${ }^{1} \mathrm{H}_{-}{ }^{15} \mathrm{~N}$ HSQC measurements (Figure 2a), respectively, in the dilute and crowded environments.

\section{References}

1. Shen, Y.; Delaglio, F.; Cornilescu, G.; Bax, A., TALOS+: a hybrid method for predicting protein backbone torsion angles from NMR chemical shifts. J. Biomol. NMR 2009, 44 (4), 213-223. 